\title{
HOMOGENEOUS NEWTONIAN COSMOLOGIES AND THEIR PERTURBATIONS
}

\author{
PETER SZEKERES and JOHN R. RANKIN
}

(Received 4 January 1977)

\begin{abstract}
A review of Heckmann and Schücking's formulation of Newtonian cosmology is presented, which permits the discussion of models more general than those possessing both homogeneity and isotropy. In particular it is shown that all homogeneous cosmologies may be uniquely specified by the rate of shear tensor as an arbitrary function of time and specifying arbitrary initial values for expansion, rotation and density. Perturbations of these models are now discussed, with a view to their possible implications for galaxy formation. The Jeans criterion is shown to hold in all these models, even in the presence of viscosity; this generalizes a result of Bonnor which only applied to the isotropic case. Furthermore, Bonnor's analysis is considerably simplified in the present paper. Finally, a WKB-type of approximation procedure is described which appears to be successful in estimating the growth rate of unstable fluctuations.
\end{abstract}

\section{Introduction}

Cosmology, the study of the gross dynamical behaviour of the universe, is generally regarded as being the province of general relativity. However, the cosmological equations are in general difficult to solve, and once a cosmological solution has been found its stability poses yet another equally interesting and challenging problem. The most famous and frequently used class of solutions are those representing isotropic and homogeneous solutions, and are known as Friedmann models. The perturbation theory of these models was analysed by Lifschitz [1] in 1946, and he came to the conclusion that the Jeans stability criterion [2] was valid for this class of models, but the rate of growth followed a power law rather than an exponential one, so that the suitability for galaxy formation (that is sizeable inhomogeneities) became questionable. Nevertheless, whether one accepts or rejects the hypothesis that galaxies form from perturbations in a homogeneous background, the existence of the Jeans wavelength as a natural scaling length on the size of unstable fluctuations remains a powerful idea, the relevance of which it seems premature to abandon out of hand.

A question of considerable interest is then whether the Jeans criterion continues to hold good in more general backgrounds, such as models possessing rotation and 
shear. It is with this aim in mind that we turn our attention in this paper to cosmologies in the simpler context of Newtonian gravitation theory. That the Friedmann models have a simple Newtonian interpretation has been known for some time [3,4], and their perturbation theory was shown by Bonnor [5] to be essentially equivalent to Lifschitz's analysis while being correspondingly simpler. However, less well known is the formulation of Newtonian cosmology given by Heckmann and Schücking [6], which permits discussion of non-isotropic models. As their original work is in German, has received scant attention in the literature and is a profitable field for comparison with general relativistic models, we feel this to be a suitable moment to review some of their basic results. This is done in Sections 2, 3 and 4 of this paper.

Our formulation of Newtonian cosmology in Section 2 essentially follows the Heckmann-Schücking model, with minor departures on detail and interpretation. In Section 3 we give a definition of what we mean by a "homogeneous" cosmology, and prove their somewhat surprising result [7] that a homogeneous cosmology is uniquely specified by five arbitrary functions of time (namely, the rate of shear) and five arbitrary constants (initial values of the density, expansion and angular velocity). In Section 4 we concentrate on shear-free models, and show that with rotation present all such models "bounce" out of the singularity which inevitably occurs in the isotropic (rotation-free) case. These results are compared and contrasted with what is now known to be the case in general relativity.

In Section 5 we set up the perturbation analysis, and show that the "comoving" Fourier components have a time development given by a set of ordinary differential equations. The stability analysis shows that the Jeans criterion is valid for the general homogeneous cosmology, even if viscosity is included. Some special cases, where the density growth equation reduces to a single second-order differential equation, are discussed in Section 6 . These include the Friedmann case, the shearfree rotating case with perturbations along or perpendicular to the axis of rotation, and some non-rotating shearing models with initially stationary perturbations. Finally, a WKB-type approximation procedure for evaluating the rate of growth of perturbations is discussed in Section 7. It is shown to converge rigorously to the correct rate in the Einstein-de Sitter model.

\section{Newtonian cosmology}

Following Heckmann and Schücking [6] we shall understand by a Newtonian cosmology, the following: a three-dimensional Euclidean space, whose points are denoted by position vectors $\mathbf{r}$, a time parameter $t$, three functions of space and time $\rho(\mathbf{r}, t), p(\mathbf{r}, t), \phi(\mathbf{r}, t)$ called respectively the density, pressure and gravitational potential, and a vector field $\mathbf{v}(\mathbf{r}, t)$ known as the relocity field, related by the standard 
equations of fluid dynamics

$$
\begin{gathered}
\frac{d \rho}{d t}+\rho \nabla \cdot \mathbf{v}=0, \\
\frac{d}{d t} \mathbf{v}=-\nabla \phi-\frac{1}{\rho} \nabla p, \\
\nabla^{2} \phi=4 \pi G_{\rho},
\end{gathered}
$$

where $d / d t \equiv \partial / \partial t+\mathbf{v} . \nabla$. For simplicity reference to a cosmological constant has been omitted here, but it could easily be included by inserting a term $-\Lambda$ on the right-hand side of equation (3).

By a fundamental observer we mean any streamline $\mathbf{r}(t)$ of the motion, that is a solution of the differential equation

$$
\frac{d \mathbf{r}}{d t}=\mathbf{v}(\mathbf{r}(t), t)
$$

Newtonian physics depends heavily on the notion of inertial observers. These find no natural place in the above scheme, and indeed the task of combining the notion of an inertial frame and an infinite (non-empty) cosmology appears to be essentially impossible $[4,8,9]$. The heart of the difficulty may be summed up as follows. Equations (1)-(3) are invariant under the Galilean group of coordinate transformations

$$
\begin{gathered}
t^{\prime}=t+t_{0} \\
x_{i}^{\prime}=a_{i j} x_{j}+u_{i} t+b_{i}, \quad a_{i j} a_{i k}=\delta_{j k}
\end{gathered}
$$

if we assume that $\rho, p$ and $\phi$ are scalar fields,

$$
\rho^{\prime}\left(\mathbf{r}^{\prime}, t^{\prime}\right)=\rho(\mathbf{r}, t) \text {, et cetera, }
$$

and the components of velocity transform as a vector field

$$
v_{i}^{\prime}\left(\mathrm{r}^{\prime}, t^{\prime}\right)=a_{i j} v_{j}(\mathbf{r}, t)+u_{i} .
$$

Thus the dynamical equations look the same in all inertial frames. However, the cosmology (that is the solution of these equations) is certainly not invariant under these transformations, for the functional forms of $\rho, p, \phi$ and $\mathbf{v}$ will be quite different in different frames. Furthermore, the notion of an inertial particle finds no natural place in our formulation, for presumably a "free" particle has equation of motion

$$
\ddot{\mathbf{r}}=-\nabla \phi,
$$

and rectilinear motion only results if $\phi=$ const, whence $\rho=0$ by equation (3). Thus the word "Newtonian" only refers here to the form of the field equations, not to any fundamental questions of dynamics. 
It should also be noted that no sensible equations for light propagation can be postulated, so that our cosmologies are essentially dark. No attempt should then be made to assign any observational consequences to the models which result. Dynamically, however, there is room for comparison with general relativistic models.

\section{Homogeneous Newtonian cosmologies}

Let $\mathbf{a}(t)$ be any fundamental observer. Relative to a we may define the position and velocity of any other point $\mathbf{r}$ at time $t$ as being

$$
\begin{aligned}
\mathbf{r}_{a} & =\mathbf{r}-\mathbf{a}(t), \\
\mathbf{v}_{a} & =\mathbf{v}(\mathbf{r}, t)-\mathbf{v}(\mathbf{a}(t), t) \\
& =\mathbf{v}\left(\mathbf{r}_{a}+\mathbf{a}(t), t\right)-\mathbf{v}(\mathbf{a}(t), t) \\
& =\mathbf{v}_{a}\left(\mathbf{r}_{a}, t\right) .
\end{aligned}
$$

The vector function $\mathbf{v}_{a}(x, t)$ so defined is called the velocity field relative to $\mathbf{a}(t)$.

We will say that the cosmology is homogeneous if the density and pressure are independent of position at any time $t$, that is $\rho=\rho(t), p=p(t)$, and if the velocity field relative to all fundamental observers is the same, that is

$$
\mathbf{v}_{a}(\mathbf{x}, t)=\mathbf{v}_{b}(\mathbf{x}, t) .
$$

This equation says that

$$
\mathbf{v}(\mathbf{x}+\mathbf{a}(t), t)-\mathbf{v}(\mathbf{a}(t), t)=\mathbf{v}(\mathbf{x}+\mathbf{b}(t), t)-\mathbf{v}(\mathbf{b}(t), t),
$$

and on taking derivatives with respect to $x_{i}$ at $\mathbf{x}=\mathbf{0}$, we obtain

$$
\frac{\partial v_{i}}{\partial x_{j}}(\mathbf{a}, t)=\frac{\partial v_{i}}{\partial x_{j}}(\mathbf{b}, t) \text {. }
$$

But $\mathbf{a}$ and $\mathbf{b}$ are arbitrary at any time $t$ (assuming a fundamental observer through every spatial point), whence

$$
\begin{aligned}
v_{i, j} & =V_{i j}(t), \\
v_{i} & =V_{i j}(t) x_{j}+c_{j}(t) .
\end{aligned}
$$

On substituting into equation (2) we find that the potential gradient $\phi_{, i}$ must have the form

where

$$
\phi_{, i}=-f_{i j}(t) x_{j}-d_{i}(t),
$$

and

$$
f_{i j}=V_{i j}+V_{i k} V_{k j}
$$

$$
d_{i}=\dot{c}_{i}(t)+V_{i j} c_{j}
$$

There is little loss of generality in setting $\mathbf{c}=\mathbf{d}=\mathbf{0}$, since by going to an "accelerated" reference frame $\mathbf{r}^{\prime}=\mathbf{r}+\mathbf{g}(t)$ with $\dot{\mathbf{g}}=-\mathbf{c}$ and redefining the potential $\phi$ as $\phi^{\prime}=\phi-\left(\ddot{g}_{i}+V_{i j} \dot{g}_{j}\right) x_{i}$ all the equations (1)-(3) are again satisfied. 
Thus by a homogeneous Newtonian cosmology we shall mean one having the form

$$
\rho=p(t), \quad p=p(t), \quad v_{i}=V_{i j}(t) x_{j}, \quad \phi_{, i}=-f_{i j}(t) x_{j} .
$$

Since $\phi_{, i j}=\phi_{, j i}$ we must have that the matrix $F=\left(f_{i j}\right)$ is symmetric. The cosmological equations (1)-(3) may be written as

$$
\begin{gathered}
\dot{\rho}+\rho V_{i i}=0, \\
\dot{V}_{i j}+V_{i k} V_{k j}=f_{i j}, \\
f_{i i}=-4 \pi G \rho .
\end{gathered}
$$

Breaking up $V_{i j}$ into a trace, symmetric trace-free part and skew part (that is the irreducible parts under the action of the rotation group)

$$
V_{i j}=\frac{1}{3} \theta \delta_{i j}+\sigma_{i j}+\omega_{i j}
$$

where

$$
\begin{aligned}
\theta & =V_{i i}=\text { expansion, } \\
\sigma_{i j} & =\frac{1}{2}\left(V_{i j}+V_{j i}\right)-\frac{1}{3} \theta \delta_{i j}=\text { shear, } \\
\omega_{i j} & =\varepsilon_{j i k} \omega_{k}=\frac{1}{2}\left(V_{i j}-V_{j i}\right)=\text { rotation, }
\end{aligned}
$$

we find that $(8)$ reduces to three equations

$$
\begin{aligned}
\dot{\theta} & =-4 \pi G \rho-\frac{1}{3} \theta^{2}+2 \omega^{2}-2 \sigma^{2}, \\
\dot{\omega}_{i} & =-\frac{2}{3} \theta \omega_{i}+\sigma_{i j} \omega_{j}, \\
\dot{\sigma}_{i j} & =-\frac{2}{3} \theta \sigma_{i j}-\sigma_{i k} \sigma_{k j}-\omega_{i k} \omega_{k j}+\frac{2}{3}\left(\sigma^{2}-\omega^{2}\right) \delta_{i j}+f_{i j}-\frac{1}{3} f_{k k} \delta_{i j},
\end{aligned}
$$

where

$$
\begin{aligned}
& \sigma^{2}=\frac{1}{2} \sigma_{i j} \sigma_{i j}, \\
& \omega^{2}=\frac{1}{2} \omega_{i j} \omega_{i j}=\omega_{i} \omega_{i}=\omega^{2} .
\end{aligned}
$$

The structure of these equations is fairly clear. Given an arbitrary shear matrix $\sigma_{i j}(t)$ as a function of time, we may solve equations (7), (10) and (11) for $\theta, \omega_{i}$ and $\rho$, a unique solution resulting for every set of initial values of these variables. Finally, the trace-free part of $f_{i j}$ is read off from equation (12), and its trace from equation (9). Thus the set of homogeneous Newtonian universes is determined by five arbitrary functions of time (the rate of shear) and five arbitrary constants (the initial values of $\omega_{i}, \rho$ and $\phi$ at an arbitrary time $t_{0}$ ). It is interesting to note how different this situation is from general relativity where there are nine possible groups of symmetry for a universe with homogeneous space sections (the Bianchi types), and each one must be treated separately [10]. 


\section{Shear-free universes}

The easiest case to analyse is when we set

$$
\sigma_{i j}(t)=0 \text {. }
$$

If we introduce a function $R(t)$ defined by

$$
\theta=3 \frac{\dot{R}}{R}
$$

then equations (7) and (11) result in

$$
\begin{aligned}
& \rho=\rho_{0} R^{-3}, \\
& \omega=\omega_{0} R^{-2}
\end{aligned}
$$

with $\rho_{0}, \omega_{0}$ constants.

Finally, equation (10) gives

$$
\frac{\ddot{R}}{R}=-\frac{4 \pi G}{3} \frac{\rho_{0}}{R^{3}}+\frac{2 \omega_{0}^{2}}{R^{4}}
$$

which integrates out to give the Heckmann-Schücking equation [7]

$$
\dot{R}^{2}=\frac{8 \pi G \rho_{0}}{3 R}-\frac{2 \omega_{0}^{2}}{3 R^{2}}-\frac{\varepsilon}{\tau_{0}^{2}}=f(R),
$$

where $\varepsilon= \pm 1$ or $0, \tau_{0}$ an arbitrary constant.

The case $\omega_{0}=0$ is the shear-free, rotation-free case (isotropic) and has a strong analogy with the Friedmann models of general relativity [4], even down to the precise form of the dynamical equation (13).

However, with $\omega_{0} \neq 0$, the models have no apparent analogies in general relativity, for a theorem of Schücking [11] tells us that all homogeneous shear-free dust solutions $(p=0)$ of Einstein's equations must have either vanishing expansion (that is, are stationary) or vanishing rotation (that is, are Friedmann models). The theorem has been shown by Ellis [12] to hold even in the inhomogeneous case. The stationary models have a Newtonian counterpart, on setting $\theta=0, \sigma=0$ and after a suitable orientation of axes

$$
\rho=\text { const }, \quad \omega=[0,0, \sqrt{ }(2 \pi G \rho)], \quad \phi=-\pi G \rho\left(x^{2}+y^{2}\right) .
$$

The rotating expanding models defined by equation (13) can have no general relativistic counterpart, by the Schücking-Ellis theorem. They have the interesting feature that they all bounce, attaining some minimum "radius" $R_{0}$ where $f\left(R_{0}\right)=0$, no matter how small a rotation $\omega_{0}$ is imparted to the model. For $\varepsilon=+1$, a maximum radius $R_{1}$ is attained, the solution oscillating forever between the two values $R_{0}$ and $R_{1}$, while for $\varepsilon=0$ or -1 the solutions contract from infinity to $R_{0}$ then expand back to infinity. All such singularity-free models are now known to be impossible in general relativity as a consequence of powerful theorems of Hawking 
and Penrose [13]. However, they imply that an original hypothesis of Lifschitz and Khalatnikov [14] concerning the possibility of a bounce being induced by rotation is valid for Newtonian models. It leads us to speculate on whether the hypothesis might not be valid in some weaker form of general relativity, for example by hypothesizing that in some sense to be defined, almost all geodesics can be made to escape the singularity in rotating models.

It should, however, be pointed out that the Heckmann-Schücking formulation of Newtonian cosmology is by no means universally accepted. For example, in a version adopted by Davidson and Evans [15] none of the above conclusions follow. Their assumptions amount to assuming that the gravitational field is isotropic, that is

$$
f_{i j}=-\frac{4}{3} \pi G \rho \delta_{i j}
$$

in our terminology. Then within this restricted class of cosmologies they show that all models have singularities and that $\sigma_{i j}=0$ implies $\theta \omega=0$ just as in general relativity. However, the reasons for these results holding are very different from those in general relativity, and the absence of anisotropic components of $f_{i j}$ is equivalent to no "free" gravitational field, or Weyl tensor, in general relativity. As this seems too strong a condition to impose, and is one which must of necessity be abandoned in the perturbation theory which follows, we shall not adopt this procedure here.

\section{Comoving perturbations}

In this section we discuss the theory of perturbations of the above models, following a method originally used by Bonnor [5] for the isotropic case.

Consider a small perturbation of a Newtonian model

$$
\rho \rightarrow \rho+\delta \rho, \quad p \rightarrow p+\delta p, \quad \phi \rightarrow \phi+\delta \phi, \quad \mathbf{v} \rightarrow \mathbf{v}+\delta \mathbf{v} .
$$

We will assume that $p$ and $\rho$ are connected by an equation of state

such that

$$
p=p(\rho)
$$

$$
\begin{gathered}
\delta p=\frac{d p}{d \rho} \delta p \equiv c_{s}^{2} \delta \rho \\
\left(c_{s}=\sqrt{ }(d p / d \rho)=\text { speed of sound }\right) .
\end{gathered}
$$

The perturbed equations (1)-(3) give

$$
\begin{gathered}
\frac{d}{d t}(\delta \rho / \rho)+\nabla . \delta \mathbf{v}+\frac{1}{\rho} \nabla \rho . \delta \mathbf{v}=0 \\
\frac{d}{d t} \delta \mathbf{v}+(\delta \mathbf{v} . \nabla) \mathbf{v}=-\nabla \delta \phi-c_{g}^{2} \nabla(\delta \rho / \rho)-\left(\frac{d^{2} p}{d \rho^{2}} \nabla \rho\right)(\delta \rho / \rho), \\
\nabla^{2} \delta \phi=4 \pi G \rho(\delta \rho / \rho) .
\end{gathered}
$$


Bonnor's method of treating these equations is essentially the following. Consider "comoving" Fourier components of the perturbed quantities

$$
\begin{aligned}
\delta \rho / \rho & =h(t) \exp (i \mathbf{k}(t) . \mathbf{r}), \\
\delta \mathbf{v} & =i \mathbf{u}(t) \exp (i \mathbf{k}(t) . \mathbf{r}), \\
\delta \phi & =\psi(t) \exp (i \mathbf{k}(t) . \mathbf{r}),
\end{aligned}
$$

where $\mathbf{k}(t)$ is chosen such that the phase of the waves are "frozen" in the cosmic fluid

$$
\frac{d}{d t}(\mathbf{k}(t) \cdot \mathbf{r})=0 .
$$

For homogeneous models this is always possible, $\mathbf{k}(t)$ being any solution of the differential equation

$$
k_{i}+V_{j i} k_{j}=0 \text {. }
$$

The technique may be extendible to short wavelength perturbations (that is $|\mathbf{k}| \gg|\nabla \rho / \rho|)$ in inhomogeneous models if we permit $\mathbf{k}$ to be a function of both $t$ and $\mathbf{r}$.

It is now a straightforward matter to substitute these perturbations into (14)-(16) for the homogeneous case $(\nabla \rho=0)$, to obtain

$$
\begin{gathered}
h-k_{i} u_{i}=0, \\
\dot{u}_{i}+V_{i j} u_{j}+k_{i}\left(\psi+c_{s}^{2} h\right)=0, \\
k^{2} \psi+4 \pi G \rho h=0 .
\end{gathered}
$$

Using (17) we find on differentiating (18a)

$$
h+2 k_{i} V_{i j} u_{j}+\left(k^{2} c_{s}^{2}-4 \pi G \rho\right) h=0 .
$$

If at $t=t_{0}, h=h_{0}>0, \mathbf{u}_{0}=0$, then from (18a) we have $h_{0}=0$ and from (19)

$$
h_{0}=-\left(k_{0}^{2} c_{s}^{2}-4 \pi G \rho_{0}\right) h_{0},
$$

whence $h_{0}<0$ if and only if

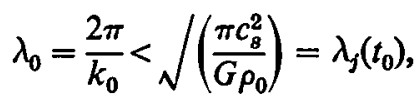

the standard Jeans criterion for stability.

It is worth noting here that the extreme generality of the above result does not seem to be altered by including the effects of viscosity into our cosmology. The fundamental cosmological equations should in this instance be altered by the addition of terms

$$
\nu_{1} \nabla^{2} \mathbf{v}+\left(\frac{1}{3} \nu_{1}+v_{2}\right) \nabla(\nabla . v)
$$

to the right-hand side of equation (2). $\nu_{1}$ and $\nu_{2}$ are the first and second coefficients of viscosity; the resulting equation is the well-known Navier-Stokes equation. It 
clearly has no effect on homogeneous solutions since in these $\mathbf{v}$ is a linear function of $\mathbf{r}$. For homogeneous cosmologies, $\nu_{1}$ and $\nu_{2}$ will be at most (known) functions of time and the above perturbation analysis may again be carried out, equation (19) becoming modified to read

$$
\grave{h}+2 V_{i j} k_{i} u_{j}+\left(\frac{4}{3} \nu_{1}+\nu_{2}\right) k^{2} h+\left(c_{s}^{2} k^{2}-4 \pi G \rho\right) h=0 .
$$

Thus viscosity does not alter the Jeans criterion for stability, but it will act as a damping term, causing unstable perturbations to grow at a slower rate.

\section{Special cases}

The rate of growth of perturbations is governed by a set of ordinary differential equations (18a)-(18c) and (17), which it is difficult to analyse in detail. There are, however, a few simple cases, which we now give, in which the rate of growth of the fluctuation contrast density is governed by a single second order differential equation.

(i) Friedmann models, $\omega=\sigma=0$

In this case equation (19) reduces immediately to the second-order BonnorLifschitz equation $[1,5]$

$$
\ddot{h}+2 \frac{\dot{R}}{R} h+\left(k^{2} c_{s}^{2}-4 \pi G \rho\right) h=0 .
$$

Integration of equation (17) gives immediately that $k=\mathbf{k}_{0} R^{-1}$, thus the wavelength of these perturbations grows with $R(t)$ just as expected for comoving perturbations. On the other hand, $\rho=\rho_{0} R^{-3}$ so that the Jeans length $\lambda_{j}$ may be expected to grow as $R^{t} c_{s}$. Thus the question whether an initially unstable perturbation remains unstable or will eventually stabilize depends on the nature of the function $R^{t} c_{s}(t)$, namely on the time dependence of the speed of sound $c_{s}$.

A particularly simple case is the Einstein-de Sitter model found by setting $\varepsilon=\omega_{0}=0$ in equation (13), to give

$$
R \propto t^{\ddagger}, \quad 4 \pi G \rho=\frac{2}{3} t^{-2} .
$$

For cold perturbations ( $\delta p=0, c_{s}=0$ ), equation (20) reduces immediately

$$
\grave{h}+\frac{4}{3 t} h-\frac{2}{3 t^{2}} h=0 \text {, }
$$

the solution to which is easily seen to be

$$
h=h_{0} t^{-1}+h_{1} t^{t}
$$

Thus the unstable perturbations grow as $t$. Such a growth law appears to be quite inadequate to account for the formation of galaxies with contrast densities of the order of $10^{6}$, particularly if the initial fluctuations are taken to be thermal. 
(ii) Shear-free homogeneous models

As derived previously in Section 4

$$
\dot{\omega}=-2 \frac{\dot{R}}{R} \omega, \quad \omega=\omega_{0} R^{-2} .
$$

Equation (17) gives

$$
\mathbf{k}=-\frac{\dot{R}}{R} \mathbf{k}-\mathbf{k} \times \omega,
$$

whence

$$
(\omega . \mathrm{k}) \cdot=-3 \frac{\dot{R}}{R}(\omega . \mathrm{k}), \quad \omega . \mathrm{k}=\omega_{0} \mathrm{k}_{0} R^{-3}
$$

and

$$
(\mathrm{k} \times \omega)^{\cdot}=-3 \frac{\dot{R}}{R}(\mathrm{k} \times \omega)+\omega \times(\mathrm{k} \times \omega)
$$

On the other hand, equation (19) reduces in this case to

$$
\grave{h}+\frac{2 \dot{R}}{R} h+\left(k^{2} c_{s}^{2}-4 \pi G \rho\right) h+2 \mathbf{u} . \mathbf{k} \times \omega=0
$$

and also we obtain

$$
(\mathbf{u} . \mathbf{k} \times \omega)^{\cdot}=-\frac{4 \dot{R}}{R} \mathbf{u} . \mathbf{k} \times \omega+2(\mathbf{u} \times \omega) .(\mathbf{k} \times \omega) .
$$

Now if initially $\mathbf{k} \times \boldsymbol{\omega}=\mathbf{0}$, that is $\mathbf{k}$ is parallel to the rotation vector $\boldsymbol{\omega}$, then by (22) it remains so, hence the last term in (23) reduces to zero, and we are back to the Bonnor-Lifschitz equation (20).

The most general case is hard to analyse, but if initially $\omega . k=0$, then by (21) $k$ remains perpendicular to $\omega$, hence

$$
(\mathbf{u} . \mathbf{k} \times \omega)^{\cdot}=-\frac{4 \dot{R}}{R} \mathbf{u} . \mathbf{k} \times \omega+\frac{2 \omega_{0}}{R^{4}} h
$$

which integrates to give

$$
\mathbf{u} . \mathbf{k} \times \omega=2 \omega_{0}^{2}\left(h-h_{1}\right) R^{-4}, \quad h_{1}=\text { const }
$$

and (23) gives a generalized Bonnor-Lifschitz equation

$$
\ddot{h}+\frac{2 \dot{R}}{R} h+\left(k^{2} c_{s}^{2}-4 \pi G \rho+4 \omega_{0}^{2} R^{-4}\right) h-4 \omega_{0}^{2} h_{1} R^{-4}=0 .
$$

Thus rotation has a small stabilizing effect on perturbations orthogonal to $\omega$, for it makes the coefficient of $h$ more positive. The easiest way to understand this stabilizing effect is to consider perturbations of a static universe [16], $R=1, R=0$, $\rho=$ const. In this case an initial perturbation $h_{0}>0, \mathrm{u}_{0}=0$ which is stable with respect to $\omega=0$ remains stable when we set $\omega>0$, but its period of oscillation is 
increased. If such an initial perturbation is unstable with respect to $\omega=0$, that is if $k^{2} c_{s}^{2}-4 \pi G \rho<0$, then it will remain unstable if $\omega$ is small enough but with diminished growth rate, while if $\omega$ is large enough it eventually stabilizes the perturbation by causing $h(t)$ to oscillate between two positive values of which $h_{0}$ is the minimum. Such a situation might be termed metastable; it is initially unstable but rotation acts as a secular stabilizer.

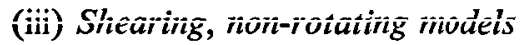

Consider models having $\omega=0$, and $\sigma_{i j}(t)$ diagonal, that is such that the principal shear axes remain fixed in direction. In this case we may set

$$
V_{i j}=\frac{\dot{R}_{i}}{R_{i}} \delta_{i j} \quad \text { (no summation on } i \text { ) }
$$

and equations (7), (8) and (9) imply

$$
\begin{gathered}
\rho=\rho_{0} / R_{1} R_{2} R_{3}, \\
\sum_{i} \ddot{R}_{i} / R_{i}=-4 \pi G \rho .
\end{gathered}
$$

Now for a comoving perturbation equation (17) gives at once that

$$
k_{i}=k_{i 0} R_{i}^{-1}
$$

and equation (18b) integrates to give

$$
u_{j}=-k_{j}\left(\int_{t_{0}}^{t} v_{J}^{2} h d t+a_{j}\right), \quad v_{J}^{2}=c_{s}^{2}-4 \pi G_{\rho} / k^{2} .
$$

Hence if initially at $t=t_{0}$, the perturbation is stationary, $\mathbf{u}_{0}=0$, we may set $a_{j}=0$ and substituting into equation (19) we obtain another Bonnor-Lifschitz equation

$$
\ddot{h}-\frac{2 k}{k} h+v_{J}^{2} k^{2} h=0, \quad k^{2}=\Sigma k_{i}^{2}
$$

(Note this equation is also valid for arbitrary shear with $k_{i}$ satisfying equation (17).) Thus for cold perturbations along a principal axis, say $\mathbf{k}_{0}=\left(k_{0}, 0,0\right)$,

$$
\grave{h}+\frac{2 \dot{R}_{1}}{R_{1}} h-4 \pi G \rho h=0 .
$$

For simplicity let us consider a power law time development

$$
R_{i}=\left(t / t_{0}\right)^{p_{i}} \text {. }
$$

Then equations (25) and (26) imply that

$$
\sum_{i} p_{i}=2
$$

and

$$
4 \pi G \rho=\alpha t^{-2}, \quad \alpha=2-\sum_{i} p_{i}^{2}
$$


Thus for positive density we must have

$$
\sum p_{i}^{2}<2 \text {. }
$$

When combined with equation (28) it is easy to show that this inequality implies that

Equation (27) has the solution

$$
0<p_{i}<\frac{4}{3}
$$

where

$$
h=h_{0} t^{a},
$$

$$
a=\frac{1}{2}\left(1-2 p_{1} \pm \sqrt{ }\left[\left(1-2 p_{1}\right)^{2}+4 \alpha\right]\right) .
$$

Now the maximum value of $\alpha$ is readily seen to occur for $p_{2}=p_{3}=1-\frac{1}{2} p_{1}$, whence

$$
\max a=\frac{1}{2}\left[1-2 p_{1}+\sqrt{ }\left(1+4 p_{1}-2 p_{1}^{2}\right)\right]
$$

and the maximum possible rate of growth of $h$ occurs when

$$
p_{1}=0, \quad p_{2}=p_{3}=1, h=h_{0} t^{a} .
$$

Thus shear does not appear to significantly modify the rate of growth of perturbations.

\section{Estimation of growth law}

Given an arbitrary homogeneous cosmology, with $V_{i j}(t)$ a specified function of $t$, it is in general quite difficult to estimate the rate of growth of the most unstable mode of a density perturbation. Here we give an effective procedure for doing this by a sequence of approximations reminiscent of the WKB technique. Consider an arbitrary comoving perturbation having wave vector $\mathbf{k}(t)$, which must be a solution of equation (17). Clearly such a mode is uniquely specified by giving the direction $\mathbf{k}_{\mathbf{0}}$ (arbitrarily) at some initial time $t=t_{0}$. Setting

$$
\begin{gathered}
h(t)=h_{0} \exp \left(\int \alpha(t) d t\right), \quad h_{0}=\text { const, } \\
u_{i}(t)=w_{i}(t) \exp \left(\int \alpha(t) d t\right),
\end{gathered}
$$

we obtain, on substitution into the perturbation equations (18), the pair of equations

$$
\begin{gathered}
\alpha h_{0}-k_{j} w_{j}=0, \\
\dot{w}_{i}+k_{i} v_{J}^{2} h_{0}+\left(\alpha \delta_{i j}+V_{i j}\right) w_{j}=0 .
\end{gathered}
$$

As a zeroth approximation we try "eikonal" perturbations, $w_{i}(t)=$ const. Then $\alpha$ must be a root of the eigenvalue equation

$$
\left|\begin{array}{cc}
\alpha & -k_{j} \\
k_{i} v_{J}^{2} & \alpha \delta_{i j}+V_{i j}
\end{array}\right|=0 .
$$


Let $\alpha_{(0)}(t)$ be the root of this equation with largest real part (if $v_{J}^{2}$ is negative and $V_{i j}$ is "reasonable" in a sense which it is difficult to specify precisely, then it may be assumed that this real part will be positive). Let us set the corresponding eigenvector

$$
\mathbf{w}_{(0)}=\mathbf{y}_{(0)}(t) h_{0} \text {. }
$$

Substituting this into the first term of equation (30) we proceed to the first approximation $\alpha_{(1)}(t)$, this being the roor with iargest reai part of the eigenvaiue equation

$$
\left|\begin{array}{cc}
\alpha & -k_{j} \\
\dot{y}_{i(0)}+k_{i} v_{J}^{2} & \alpha \delta_{i j}+V_{i j}
\end{array}\right|=0 .
$$

This provides a new value for $w_{j}$, and again we set

$$
\mathbf{w}_{(1)}=\mathbf{y}_{(1)}(t) h_{0}
$$

which we feed back into equation (30). In this way we generate a well-defined sequence $\alpha_{(0)}(t), \alpha_{(1)}(t), \ldots, \alpha_{(n)}(t), \ldots$ which hopefully converges to a definite growth function $\alpha(t)$.

Although difficult to justify rigorously, this procedure does converge rapidly to the correct growth law for the Einstein-de Sitter model, as the following analysis shows. As in Section 6(i), we take

$$
\begin{gathered}
V_{i j}=\left(\frac{2}{3} t\right) \delta_{i j}, \quad \rho=1 / 6 \pi G t^{2}, \quad \mathbf{k}(t)=\mathbf{k}_{0}\left(t / t_{0}\right)^{-\mathbf{l}}, \\
v_{J}^{2}=-\frac{2}{3} k_{0}^{2} t_{0}^{*} t^{3} \quad \text { (that is } c_{s}=0 \text { ). }
\end{gathered}
$$

Thus setting $\mathbf{k}_{0}=\left(k_{0}, 0,0\right)$, it is easily verified that the zeroth approximation gives

$$
\alpha_{(0)}\left(\alpha_{(0)}+\frac{2}{3 t}\right)-\frac{2}{3 t^{2}}=0
$$

that is

$$
\alpha_{(0)}=a_{0} / t, \quad a_{0}=(-1+\sqrt{7}) / 3 .
$$

Furthermore, the eigenvector to be fed back into the next approximation is given by

where

$$
\mathbf{y}_{(0)}=b_{0} \mathbf{k}_{0} / t^{\mathbf{t}} t_{0}^{\mathbf{j}} k_{0}^{2}
$$

$$
b_{0}\left(a_{0}+\frac{2}{3}\right)-\frac{2}{3}=0 .
$$

Successive approximations result in

$$
\alpha_{(n)}=a_{n} / t, \quad \mathbf{y}_{(n)}=b_{n} \mathbf{k}_{0} / t^{\mathbf{t}} t_{0}^{\mathbf{n}} k_{0}^{2},
$$

where the $n$th eigenvalue equation gives

$$
a_{n}\left(a_{n}+\frac{2}{3}\right)-\left(2+b_{n-1}\right) / 3=0
$$


and the corresponding eigenvector equation gives

$$
b_{n}\left(a_{n}+\frac{2}{3}\right)-\left(2+b_{n-1}\right) / 3=0 .
$$

Combining these two we see that $b_{n}=a_{n}$, and $a_{n}$ therefore satisfies the recurrence relation

$$
a_{n}\left(a_{n}+\frac{2}{3}\right)-\left(2+a_{n-1}\right) / 3=0 .
$$

If we pick the positive root at each step this is easily seen to converge to the value $a$ satisfying the quadratic

that is

$$
a^{2}+\frac{1}{3} a-\frac{2}{3}=0
$$

$$
a=\frac{2}{3}
$$

as required. The other root $a=-1$ is the limit of the negative roots, so that the stable mode is also obtainable by this procedure.

The rapidity of convergence may be seen by computing the first few terms

$$
a_{0}=0.5486, \quad a_{1}=0.6468, \quad a_{2}=0.6633, \quad a_{3}=0.6661 \quad(a=0.6667) .
$$

The success of the method in this example gives grounds for optimism that it provides an effective computational method for the growth rate of fluctuations in more general homogeneous models possessing rotation and shear. As all steps in the method are purely algebraic it is ideally suited for performing computer calculations.

\section{Acknowledgement}

One of us (P. S.) would like to express his appreciation to Professor W. B. Bonnor for an illuminating discussion concerning earlier work on these lines, and to the Mathematical Institute, Oxford, for their hospitality during the preparation of this paper.

\section{REFERENCES}

[1] E. M. Lifschitz, J. Phys. 10, (1946), 116.

[2] J. Jeans, Astronomy and Cosmology, pp. 345-350, Dover, New York (1961).

[3] E. A. Milne and W. H. McRae, Quart. J. Math. Oxford Ser. 5 (1934), 64, 73.

[4] H. Bondi, Cosmology, Cambridge University Press (1958).

[5] W. B. Bonnor, Mon. Not. Roy. Astron. Soc. 117 (1957), 104.

[6] O. Heckmann and E. Schücking, Handbuch der Physik, Vol. L111, p. 489, Springer-Verlag, Berlin (1959).

[7] O. Heckmann and E. Schücking, Zeitschrift für Astrophysik 38 (1955), 95.

[8] D. Layzer, Astrophys. J. 59 (1954), 268.

[9] W. H. McRae, Astrophys. J. 60 (1954), 271.

[10] L. Shepley and I. Ryan, Homogeneous Cosmological Models, Princeton University Press (1974). 
[11] E. Schücking, Sonderdruck aus die Nat. 38 (1955), 95.

[12] G. F. Ellis, J. Math. Phys. 8 (1967), 1171.

[13] S. W. Hawking and G. F. R. Ellis, The Large Scale Structure of Space time, Cambridge University Press (1973).

[14] E. M. Lifschitz and I. M. Khalatnikov, Adv. in Phys. (Phil. Mag. Suppl.) 12 (1963), 185.

[15] W. Davidson and A. B. Evans, Int. J. Theor. Phys. 7 (1973), 353.

[16] Cf. S. Chandresekhar, Hydrodynamics and Hydromagnetic Stability, p. 591, Oxford University Press (1961).

Department of Mathematical Physics

University of Adelaide

Adelaide, S.A., 5001

Australia 